\title{
Intersubband plasmon-phonon modes in quantum wires at high temperatures
}

\author{
Marcos R. S. Tavares \\ Instituto de Física Teórica, Universidade Estadual Paulista, São Paulo, SP 01405-000, Brazil \\ (Received 5 October 2004; revised manuscript received 21 December 2004; published 29 April 2005)
}

\begin{abstract}
Coupled intersubband plasmon-phonon modes are studied in a multisubband parabolic quantum wire at room temperatures. These modes are found by calculating the spectral weight function which is related to the inelastic Raman spectra. We use a 13 subband model. The plasmon-phonon coupling strongly modifies the dispersion relation of the intersubband modes in the vicinity of the optical phonon frequency $\omega_{L O}$. Extra modes show up as a result of the electron-phonon interaction. We carefully study the density and temperature dependence of these extra modes. We also show that coupled intersubband plasmon-phonon modes should be observed for temperatures as high as $300 \mathrm{~K}$.
\end{abstract}

DOI: 10.1103/PhysRevB.71.155332

PACS number(s): 73.50.Gr, 73.21.Hb

\section{INTRODUCTION}

There has been a great deal of interest in studying manybody excitations in quasi-one-dimensional (Q1D) electron systems embedded in GaAs/AlGaAs semiconductor heterostructures. Important research results in these systems are achieved through the inelastic light (Raman) scattering such as the observation ${ }^{1}$ of Q1D collective excitations and the verification of the predicted acoustic plasmon linear dispersion relation. ${ }^{2}$ In fact, the Raman scattering turned out to be an essential technique in experimentally probing aspects of many-body excitations of fundamental importance in such systems. ${ }^{3}$ Most frequently, these systems are made of polar semiconductors where the lattice vibration induced polarization field couples to Q1D electrons and, as a consequence, a strong coupling between the intersubband plasmons and the longitudinal-optical phonons occurs whenever their frequencies match.

The electron-phonon interaction induced effects in Q1D systems were also recently studied by means of electron and phonon relaxation rates. ${ }^{4}$ These studies provide one with a very insightful information about the lifetime of both optically and thermally excited electrons (hot electrons). The calculation of this lifetime is intrinsically dependent on collective (plasmons) and single-particle (particle-hole) excitations occurring in the system and leads to an effective comprehension and control in projecting quantum wire optoelectronic applications. The plasmon-phonon coupling has also been analyzed in the so-called $\delta$-doped semiconductors, where level broadening induced effects on the Raman spectrum were shown. ${ }^{5}$

Among other issues, intersubband coupling and temperature induced effects on Q1D electron systems have also attracted much attention recently. ${ }^{6-8}$ The energy gap between subbands can be determined by the band-gap engineering, which leads to the possibility of projecting applications such as ballistic electron transistors, quantum wire-based infrared photodetectors and lasers, quantum wire THZ oscillators and modulators. Furthermore, novel applications are certainly realized at very room temperature, so that the theoretical understanding of the temperature dependence of the intersubband many-body excitations in these systems is of fundamental importance.
The electron-phonon coupling effects were also analyzed previously ${ }^{9}$ in calculating the dispersion relations of coupled intra- and intersubband plasmon-phonon modes in quantum wires at zero temperature. A three subband model was used and novel (extra) coupled intra- and intersubband modes were found around the bulk LO-phonon frequency as a result of the electron-phonon interaction. These modes arise due to the extra coupled electron-phonon collective excitations occurring in the system. They turned out to be dispersionless having a strong bulk LO-phonon character for small values of the wave vector $q$. They begin getting dispersive as they approach their correspondent single-particle excitation (SPE) continua. Still at zero temperature, the plasmon-phonon coupling was also studied ${ }^{10}$ in a quantum wire in the extreme quantum regime, i.e., when there is only one subband occupied with electrons. In this regime, no intersubband coupling induced effects are considered. A coupled plasmon-phonon mode related to electronic excitations in the single (ground) subband was found, being of zero frequency at $q=0$. An extra mode, arising as a consequence of the electron-phonon interaction, was also found being of finite frequency at $q$ $=0$ and slightly greater than the bulk LO-phonon frequency. Its physical nature turned out to be same as those appearing in Ref. 9.

We focus this paper on room temperature effects regarding coupled intersubband plasmon-phonon modes in a full multisubband Q1D system. The complete understanding of temperature induced effects in these systems is crucial in projecting novel opto-electronic devices based on polar semiconductor quantum wires which might have several occupied subbands at room temperature. In this regime, a strong intersubband coupling between the low energy subbands and the higher unoccupied subbands might significantly affect even the low-energy coupled intersubband plasmon-phonon dispersion relations. Furthermore, the question on how these coupled modes survive with increasing temperature still remains. We emphasize that they indeed survive at room temperature and could be seen in the inelastic light scattering for a given region in the $\omega-q$ plane. We study them by looking at the peaks of the calculated spectral weight function which is related to the Raman scattering. ${ }^{11}$ We use a 13 subband model in order to make sure that the dispersion relations of the low-energy coupled plasmon- 
phonon modes do not change as more subbands are included.

Our theoretical results indicate that intersubband plasmon-phonon modes should be observed in the Raman scattering for temperatures up to $300 \mathrm{~K}$. We found pronounced peaks in the spectral weight function due to the coupled intersubband plasmon-phonon mode related to electrons in the first subband. We found the whole spectral weight function getting broadened with increase temperature because of the increase in the particle-hole (single-particle) decay rates caused by the presence of thermal excitations. The boundaries of the single-particle excitation continua can no longer be realized at room temperatures because the SPE continua are thermally smeared over all the $\omega$ - $q$ plane. These excitations are then responsible for Landau damping the coupled intersubband plasmon-phonon modes. The temperature itself serves then as a broadening parameter for the collective modes at finite values of $q$. But we show that some of them survive at room temperatures for a wide range region in the $\omega-q$ plane.

The paper is organized as follows. In Sec. II we present our theoretical formulation with the working formulas. In Sec. III we provide our numerical results and discussions. We conclude in Sec. IV with a summary.

\section{THEORETICAL FORMULATION}

We consider a parabolic potential describing the confinement in the $y$ direction of a originally two-dimensional (2D) electron gas embedded into a polar semiconductor. We also assume the electron system is of zero-thickness in the $z$ direction since the energy gap between the two lowest levels due to the confinement in this direction is safely assumed to be greater than the confinement energy $\omega_{0}$ in the $y$ direction of most samples. A 13 subband model is used with a confinement frequency given by $\omega_{0}=7 \mathrm{meV}$. The energy eigenvalues of an electron in the system is then given by

$$
E_{n}\left(k_{x}\right)=\frac{\hbar^{2} k_{x}^{2}}{2 m^{*}}+\left(n-\frac{1}{2}\right) \hbar \omega_{0},
$$

where $k_{x}$ is the electron wave vector in the $x$ direction and $\left(n-\frac{1}{2}\right) \omega_{0}$ is the electron energy levels due to the confinement in the $y$ direction, with $n=1,2, \ldots$, being the subband index. We consider $\hbar=1$ throughout this paper.

Another important parameter is the localization length $y_{0}=k_{0}^{-1}=\sqrt{1 / 2 m^{*} \omega_{0}}$ of the electrons in the $y$ direction, with $m^{*}$ being the effective electron mass in the GaAs. For the confinement parameter $\omega_{0}=7 \mathrm{meV}$, both the longitudinaloptical (LO) and the transverse-optical (TO) phonon frequencies, $\omega_{L O}=36.25 \mathrm{meV}$ and $\omega_{T O}=33.29 \mathrm{meV}$, respectively, should be easily reached by the intersubband plasmon branches in the system, leading to a strong plasmon-phonon coupling around frequencies $\omega \simeq 5 \omega_{0}$. We use, therefore, bulk phonon modes throughout this work.

A safer manner to analyze collective excitations at finite temperatures in multisubband systems is to look directly at the so-called spectral weight function, ${ }^{7}$

$$
S(q, \omega)=-\sum_{\alpha \beta} \operatorname{Im}\left[\varepsilon_{b}(\omega) \chi_{\alpha \beta}(q, \omega)\right]
$$

where $\alpha \equiv\left(i, i^{\prime}\right)$ and $\beta \equiv\left(j, j^{\prime}\right)$ are the coupled indices with the subband indices $i, i^{\prime}, j$, and $j^{\prime}$. In Eq. (2), the frequency-dependent dielectric function $\varepsilon_{b}(\omega)$ models the polarization of the background polar semiconductor. It can be written as a simplified form, ${ }^{11}$

$$
\varepsilon_{b}(\omega)=1+\frac{\omega_{T O}^{2}-\omega_{L O}^{2}}{\omega^{2}-\omega_{L O}^{2}+i \omega \eta},
$$

where $\eta$ is the phenomenological parameter which has been introduced to take into account phonon mode damping effects in the crystalline structure.

The density-density correlation function $\chi_{\alpha \beta}(q, \omega)$ is obtained through the generalized random phase approximation (RPA) equation, ${ }^{11}$

$$
\sum_{l l^{\prime}} \varepsilon_{l l^{\prime}, i i^{\prime}}(q, \omega) \chi_{l l^{\prime}, j j^{\prime}}(q, \omega)=\Pi_{i i^{\prime}}(q, \omega) \delta_{i j} \delta_{i^{\prime} j^{\prime}},
$$

which involves the electronic dielectric function $\varepsilon_{\alpha \beta}(q, \omega)$ and the $1 \mathrm{D}$ multisubband irreducible polarizability,

$$
\Pi_{j j^{\prime}}(q, \omega)=2 \sum_{k_{x}} \frac{f_{j^{\prime}}\left[E_{j^{\prime}}\left(k_{x}+q\right)\right]-f_{j}\left[E_{j}\left(k_{x}\right)\right]}{E_{j^{\prime}}\left(k_{x}+q\right)-E_{j}\left(k_{x}\right)+\omega+i \gamma} .
$$

Here, $f_{j}(E)$ is the noninteracting Fermi-Dirac distribution function and $\gamma$ is the phenomenological damping constant indicating level broadening due mainly to the electron scattering by impurity centers. We take $\gamma=10^{-1} \omega_{0}$ and $\eta$ $=10^{-3} \omega_{0}$ throughout this paper, which are as realistic as those in experimental conditions. ${ }^{12}$

Single-particle excitation continua are those regions in the $\omega-q$ plane where the imaginary part of the polarizability $\operatorname{Im}[\Pi(q, \omega)] \neq 0$. These excitations are responsible for Landau damping the collective modes as they approach (or enter) their correspondent continua. ${ }^{11}$ The boundaries of these continua are well defined by doing some algebra in Eq. (5) within the clean limit, i.e., $\gamma \rightarrow 0 .{ }^{13}$ This algebra is straightforward at zero temperature, since the Fermi distribution function in Eq. (5) is given by the step function $\theta\left(E_{j}-E_{F}\right)$ with $E_{F}$ being the Fermi energy. The existence of a sharp Fermi edge leads to a well-defined curves in the $\omega$ - $q$ plane describing the boundaries of the single-particle excitation continua. Higher values of $\gamma$ should continuously broad these curves.

But the sharpness of the Fermi edge is destroyed with increasing temperature due to thermal excitations and, as a consequence, one cannot longer find the boundaries of the single-particle excitation continua by manipulating Eq. (5). ${ }^{7}$ These boundaries can no longer be realized at high temperatures because the SPE continua are thermally smeared. At finite temperature, the imaginary part of the polarizability $\operatorname{Im}[\Pi(q, \omega)] \neq 0$ at any point in the $\omega$ - $q$ plane, even in the clean limit. ${ }^{8}$ As a consequence, one has to calculate the spectral weight function concerning each plasmon-phonon mode for all values of $\omega$ and $q$ in order to study the temperature induced Landau damping in the system. As we mentioned above, spectral functions should be broadened with increas- 
ing temperature, corresponding to an increase in the singleparticle decay rates caused by the presence of thermal excitations.

On the other hand, the phenomenological parameter represented by $\gamma$ in Eq. (5) is responsible for smoothing the singular nature of the one-dimensional (1D) density of states. ${ }^{14}$ This smoothing is observed when the optical spectra is broadened by $\gamma$. It also suppresses the divergence of the plasmon emission threshold which is seen in the hot-electron energy relaxation rate. We then point out the equivalence between the temperature and the phenomenological damping parameter $\gamma$. Thermal excitation (as well as the phenomenological parameter $\gamma$ ) broadens the plasmon modes by stating that $\operatorname{Im}[\Pi(q, \omega)]$ should be finite for all values of $q$ and $\omega$. In that strict sense, they both are smoothing the singular nature of the 1D density of states. We will show below that high temperature values are responsible for strongly broadening the less weighted peaks which appear in the spectral weight function. We will also show that those peaks in the spectral weight function with great intensities survive at room temperature.

The electronic dielectric function $\varepsilon_{\alpha \beta}(q, \omega)$ in Eq. (4) is written as a summation of the two contributions: the electron-phonon interaction (first term) and the electronelectron interaction (second term). It has the following form: ${ }^{11}$

$$
\varepsilon_{\alpha \beta}(q, \omega)=\varepsilon_{b}(\omega) \delta_{\alpha \beta}-V_{\alpha \beta}(q) \cdot \Pi_{\beta}(q, \omega),
$$

where

$$
\begin{aligned}
V_{i i^{\prime}, j j^{\prime}}(q)= & \frac{2 e^{2}}{\varepsilon_{\infty}} \int d y \int d y^{\prime} \phi_{i}(y) \phi_{i^{\prime}}(y) K_{0}\left(q\left|y-y^{\prime}\right|\right) \\
& \times \phi_{j}\left(y^{\prime}\right) \phi_{j^{\prime}}\left(y^{\prime}\right)
\end{aligned}
$$

is the Coulomb electron-electron bare interaction in the 1D geometry. Here, $\varepsilon_{\infty}$ is the high-frequency dielectric constant of the background, $e$ is the electron charge, $\phi_{i}(y)$ is the single-particle wave function of the electron in the subband $i$, and $K_{0}\left(q\left|y-y^{\prime}\right|\right)$ is the zeroth-order modified Bessel function of the second order. One is allowed to write Eq. (6) provided the polarizabilities for both the electron and the phonon systems are additives when they are written within the RPA. The only assumption in its derivation is that the electrons had electron-phonon and electron-electron interactions. The electron-electron and the electron-phonon interactions are treated on the same footing. Notice that, by setting $\varepsilon_{b}(\omega)$ $=1$ in Eq. (6), i.e., without an electron-phonon interaction, the dielectric function of the Q1D electron gas within the RPA is obviously recovered. Within this approach, all temperature dependence comes out from the electron polarizability $\Pi_{\beta}(q, \omega)$ through the Fermi-Dirac function.

The symmetry of the confinement potential in the $y$ direction leads to the electron-electron Coulomb interaction,

$$
V_{\alpha \beta}(q)=0,
$$

when $i+i^{\prime}+j+j^{\prime}$ is an odd number. As a consequence, the dielectric matrix elements (both the real and the imaginary parts),

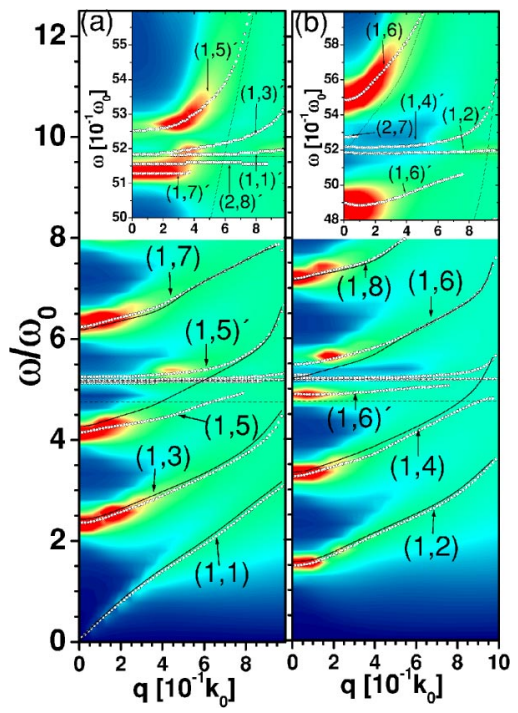

FIG. 1. (Color online) The plasmon dispersion relations obtained from the peaks in (a) $S^{\text {even }}(q, \omega)$ and (b) $S^{\text {odd }}(q, \omega)$ for $T$ $=50 \mathrm{~K}$ and $N_{e}=10^{6} \mathrm{~cm}^{-1}$. Thin solid lines show the dispersion relations without an electron-phonon interaction. The horizontal dashed lines indicate the frequencies of LO and TO phonons, $\omega_{L O}$ $=5.17 \omega_{0}$ and $\omega_{T O}=4.75 \omega_{0}$, respectively. The inset shows the vicinity of $\omega=\omega_{L O}$. The intensity scale there is changed according to the change in the $\omega$-axis scale.

$$
\varepsilon_{\alpha \beta}(q, \omega)=0,
$$

for $i+i^{\prime}+j+j^{\prime}=$ odd. The dielectric matrix can then be decoupled into two submatrices $\varepsilon_{\alpha \beta}^{\text {even }}(q, \omega)$ and $\varepsilon_{\alpha \beta}^{\text {odd }}(q, \omega)$ with both $i+i^{\prime}$ and $j+j^{\prime}$ being even and odd numbers, respectively. The even (odd) dielectric submatrix involves only that electron-electron interaction which describes the first electron scattering from subband $i$ to subband $i^{\prime}$ with $i+i^{\prime}$ $=$ even $\left(i+i^{\prime}=\right.$ odd $)$ while the second one is being scattered from subband $j$ to $j^{\prime}$ with $j+j^{\prime}=$ even $\left(j+j^{\prime}=\right.$ odd $)$. As a consequence of that, the spectral weight function can be treated separately into two parts:

$$
S(q, \omega)=S^{\text {even }}(q, \omega)+S^{\text {odd }}(q, \omega) .
$$

The peaks in the function $S(q, \omega)$ provide one with the coupled plasmon-phonon excitations and, from their $\omega$ position, one is able to obtain the dispersion relations of the modes. By showing all excitation modes through $S(q, \omega)$, one provides a very efficient guide of what should be observable in the experiments. These observations are certainly dependent on external probes (e.g., light polarization). The quantity $S(q, \omega)$ is directly related to the optical (such as inelastic light scattering spectra) and transport (such as conductivity) properties.

\section{NUMERICAL RESULTS}

We show in Fig. 1 the dispersion relations of the coupled plasmon-phonon modes (open-dot curves). They are taken from the peaks in (a) $S^{\text {even }}(q, \omega)$ and (b) $S^{\text {odd }}(q, \omega)$ for different wave vectors $q$. The temperature $T=50 \mathrm{~K}$ and the elec- 
tron density $N_{e}=1.0 \times 10^{6} \mathrm{~cm}^{-1}$. The colors indicate the intensity of the spectral weight calculated for the entire $\omega-q$ plane. They go from navy blue (dark) with zero intensity to red (soft dark), which indicates a variation of 10 times in the spectral intensity. For $N_{e}=1.0 \times 10^{6} \mathrm{~cm}^{-1}$, two subbands are occupied with the lowest one having the most electron density. This helps us to identify the most weighted peaks in Fig. 1(a): the coupled intersubband plasmon-phonon modes $(1,3)$, $(1,5)$, and $(1,7)$ as well as the intrasubband one $(1,1)$. These modes are related to electrons in the lowest subband. Extra coupled modes labeled as $(1,1)^{\prime},(1,3)^{\prime},(1,5)^{\prime}$, and $(1,7)^{\prime}$ are better seen in the inset. They show up in the vicinity of $\omega_{L O}$ as a result of the electron-phonon interaction. On the other hand, Fig. 1(b) shows the dispersion relations of the intersubband coupled modes $(1,2),(1,4),(1,6)$, and $(1,8)$. The extra modes $(1,2)^{\prime},(1,4)^{\prime}$, and $(1,6)^{\prime}$ also appear in the inset as a result of the interaction between electrons and LO phonons. These novel modes $(m, n)^{\prime}$ serve as a complement to the modes $(m, n)$ since they also correspond to coupled collective excitations involving the same subbands. They are not present in the absence of the electron-phonon interaction (solid lines). They show up due to the strong electronphonon coupling occurring in the reststrahlen region of the GaAs. Notice also that the branches above (below) $\omega_{L O}$ shift to higher (lower) frequencies when the electron-phonon interaction is considered. The presence of bulk LO phonons in the system is responsible for such a shift. This effect is also observed at zero temperature. ${ }^{9}$ The upper panels in Fig. 1 detail the vicinity of $\omega_{L O}=5.17 \omega_{0}$. The intensity scales in the upper panel are changed according to the change in the $\omega$-axis scale. In this region, only the modes related to electrons in the first subband are of noticeable intensities. But we show the less weighted extra mode $(2,8)^{\prime}$ appearing in Fig. 1(a), while the mode $(2,7)$ is seen in Fig. 1(b). We mention that these modes are being seen at $T=50 \mathrm{~K}$ due to their strong phononlike character. Notice that our model deals with bulk phonons being independent of the temperature and, as consequence, coupled modes with a strong phononlike character should be the less affected by the temperature.

At zero temperature, this system presents the second subband occupied with about $24 \%$ of the total amount of electrons. The depolarization shift (which is the energy difference between the intersubband SPE continua and its correspondent coupled intersubband mode at $q=0$ ) presented by the modes $(2, n)$ are then relatively smaller than those depolarization shifts presented by the coupled modes $(1, n)$ because there are relatively more electrons in the first subband. For the sake of clarity, we show in Fig. 2 the zerotemperature dispersion relations of the coupled plasmonphonon modes (solid curves) which are given by the zeros of the determinant of the dielectric tensors (a) $\varepsilon_{\alpha \beta}^{\mathrm{even}}(q, \omega)$ and (b) $\varepsilon_{\alpha \beta}^{\text {odd }}(q, \omega)$. The most weighted peaks appearing in (a) $S^{\text {even }}(q, \omega)$ and (b) $S^{\text {odd }}(q, \omega)$ for $T=0$ are also indicated by open circles. The upper panels show the $\omega-q$ plane around $\omega_{L O}=5.17 \omega_{0}$. Figure 2 also shows the green (dark) and yellow (bright) regions describing the single-particle excitation continua (at $T=0$ ) related to the first and second subbands, respectively. We see that depolarization shifts regarding coupled intersubband modes $(2, n)$ are about 4 times smaller

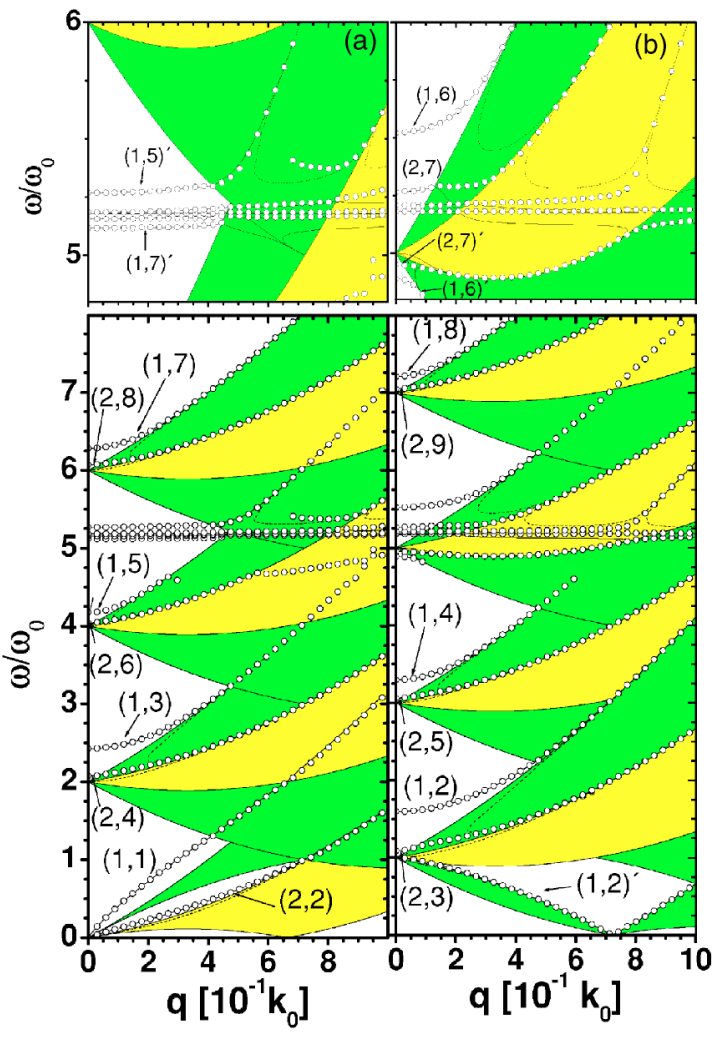

FIG. 2. (Color online) The plasmon dispersion relations at zero temperature (solid lines) obtained from the zeros of (a) $\operatorname{det}\left|\varepsilon_{\alpha \beta}^{\text {even }}(q, \omega)\right|=0$ and (b) $\operatorname{det}\left|\varepsilon_{\alpha \beta}^{\text {odd }}(q, \omega)\right|=0$. Open dots represent the plasmon dispersion relations obtained from the peaks in (a) $S^{\text {even }}(q, \omega)$ and (b) $S^{\text {odd }}(q, \omega)$. The electron density is the same as in Fig. 1. The horizontal dashed lines indicate the frequencies of LO and TO phonons, $\omega_{L O}=5.17 \omega_{0}$ and $\omega_{T O}=4.75 \omega_{0}$, respectively. The inset shows the vicinity of $\omega=\omega_{L O}$. The green (dark) and yellow (bright) regions describe the SPE continua related to the first and second subbands, respectively.

than those depolarization shifts of the modes $(1, n)$. As a consequence, the coupled modes related to electrons in the second subband are seen with much smaller intensities in the spectral weight function at $T=0$. They are then the best candidates to be affected by thermal excitation induced effects which definitely occur in the vicinity of the yellow regions.

By comparing Figs. 1 and 2, we show that most modes $(2, n)$ are indeed damped by the temperature and, as a consequence, only the coupled intersubband modes $(1, n)$ related to electrons in the first subband are seen with noticeable intensities at $T=50 \mathrm{~K}$. The temperature damps those intersubband modes which are of a small depolarization shift. Furthermore, the temperature damping on the $(1, n)$ peaks also contributes to the vanishing (damping) of those small peaks, even in the long wavelength limit. A higher spectral weight (red regions) is then found for coupled intersubband modes $(1, n)$ related to the first subband at small wave vectors $q$. Those $(2, n)$ modes not appearing in Fig. 1 have been Landau damped due to the increasing of thermal excitations in the system.

Notice that Landau damping is characterized by the energy transfer from the collective mode to particles (particle 


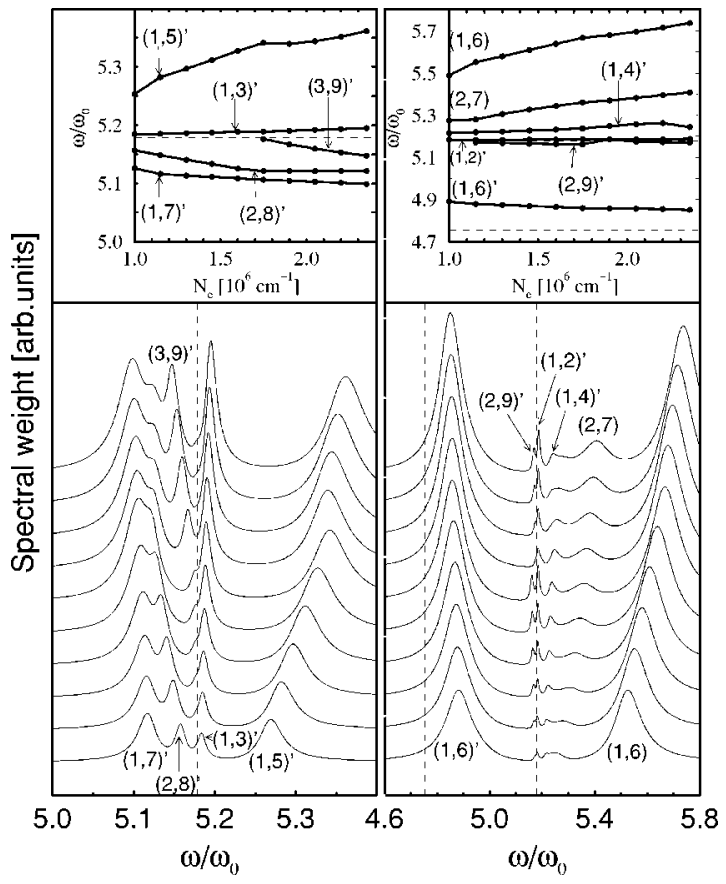

FIG. 3. The spectral weight (a) $S^{\text {even }}(q, \omega)$ and (b) $S^{\text {odd }}(q, \omega)$ for $q=0.05 k_{0}$ and $T=50 \mathrm{~K}$. The electron density varies from $N_{e}=1.0$ $\times 10^{6} \mathrm{~cm}^{-1}$ (the lowest curve) to $N_{e}=2.35 \times 10^{6} \mathrm{~cm}^{-1}$ (the top curve) with a difference of $0.15 \times 10^{6} \mathrm{~cm}^{-1}$. The insets indicate the position of the peaks as a function of $N_{e}$. Vertical and horizontal dashed lines indicate the frequencies of TO and LO phonons.

holes) traveling at the same velocity as the phase velocity of the collective modes. ${ }^{11}$ As a consequence, Landau damping at a finite temperature can be significant if the Fermi distribution function $f\left[v_{m, n}(q)\right]$ is non-negligible, with $v_{m, n}(q)$ being the phase velocity of the mode $(m, n)$. That means the Landau damping is strong if the phase velocity is less than or in the order of the thermal velocity. For instance, the coupled

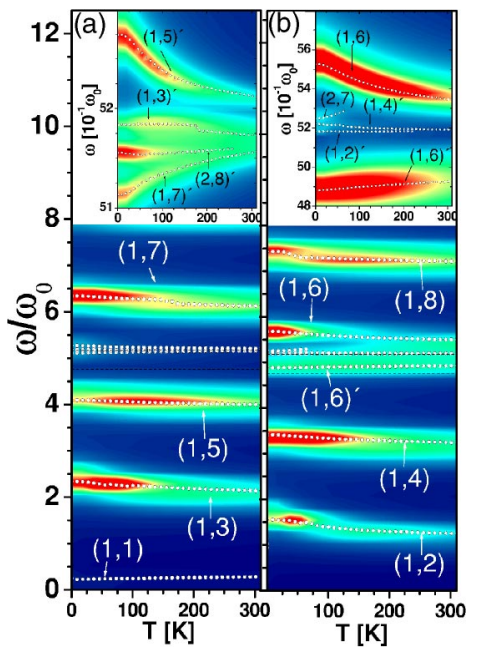

FIG. 4. (Color online) The temperature dependence of the peaks in (a) $S^{\text {even }}(q, \omega)$ and (b) $S^{\text {odd }}(q, \omega)$ for $q=0.05 k_{0}$ and $N_{e}=1.0$ $\times 10^{6} \mathrm{~cm}^{-1}$. The colors and the horizontal dashed lines indicate the same as in Fig. 1. The insets show the vicinity of the frequency $\omega=\omega_{L O}$.

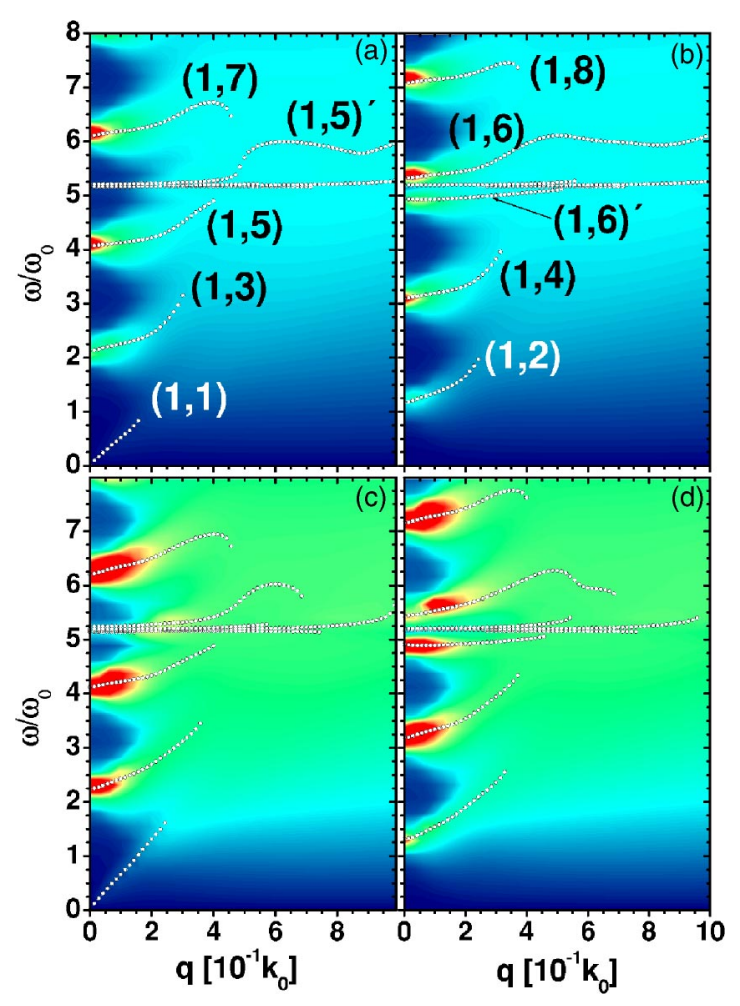

FIG. 5. (Color online) Intersubband coupled modes $(1, n)$ obtained by mapping the spectral weight function [(a) and (c)] $S^{\text {even }}(q, \omega)$ and $[(\mathrm{b})$ and $(\mathrm{d})] S^{\text {odd }}(q, \omega)$ at $T=300 \mathrm{~K}$. The electron density in (a) and (b) is $N_{e}=10^{6} \mathrm{~cm}^{-1}$, while we take $N_{e}=2.22$ $\times 10^{6} \mathrm{~cm}^{-1}$ in (c) and (d). Horizontal dashed lines indicate the frequencies of TO and LO phonons.

plasmon-phonon mode $(2,2)$ at zero temperature can be well fitted by a linear dispersion relation in the long wavelength limit. ${ }^{13}$ Its phase velocity $v_{2,2} \sim N_{2}$ is, therefore, independent of the wave vector, being mostly dependent on the electron density in the second subband $N_{2}$. Since this phase velocity does not change with increasing $q$, the thermal velocity easy reaches $v_{2,2}(q)$ at $T=50 \mathrm{~K}$, leading to the strong damping of the mode $(2,2)$, even at small $q$. In the same way, temperature induced Landau damping over the mode $(2,2)$ should also increase as the number of electrons in the second subband $N_{2}$ is reduced. Furthermore, the coupled intersubband modes $(2, n)$ present phase velocities which can be written as $v_{2, n}(q) \sim \Delta_{2, n} / q$ in the long wavelength limit, where $\Delta_{2, n}$ is the depolarization shift of the mode $(2, n) .{ }^{15}$ This depolarization shift depends directly on the electron density in the second subband. For extremely low densities in this subband, $\Delta_{2, n}$ is very small and, as a consequence, the phase velocity $v_{2, n}$ can be finite even for small values of $q$. Hence thermal velocities at room temperature can indeed reach $v_{2, n}$ and, as a consequence, the Landau damping should play its role over the intersubband modes $(2, n)$. We should mention, however, that in cases in which the phase velocities $v_{m, n}(q=0) \rightarrow \infty$ all $(m, n)$ peaks should be observed at $q=0$. But we were not able to numerically find them in Fig. 1 for $q=0$ since the temperature broadening over the $(1, n)$ peaks also contributes to the vanishing (damping) of those very small peaks in $S(q, \omega)$. 
We show in Fig. 3 the spectral weight (a) $S^{\text {even }}(q, \omega)$ and (b) $S^{\text {odd }}(q, \omega)$ as a function of frequencies around $\omega_{L O}$ for several values of the electron density $N_{e}$. The temperature is the same as in Fig. 1 and we take the wave vector $q$ $=0.05 k_{0}$. The density varies from $1.0 \times 10^{6} \mathrm{~cm}^{-1}$ (the lowest curve) to $2.35 \times 10^{6} \mathrm{~cm}^{-1}$ (the top curve) with a step of $0.15 \times 10^{6} \mathrm{~cm}^{-1}$. The spectral intensity scale in Fig. 3 is almost five times smaller than those scales indicated by the colors in Fig. 1. Intersubband modes $(1, n)$ indeed represent the most weighted peaks appearing in the spectral weight function. But we, nevertheless, keep looking closer at the vicinity of $\omega_{L O}$ where the extra modes are seen. The lowest curve in Fig. 3(a) shows the modes $(1,7)^{\prime},(2,8)^{\prime},(1,3)^{\prime}$, and $(1,5)^{\prime}$, while Fig. $3(\mathrm{~b})$ clearly shows the most weighted peaks due to the coupled intersubband plasmon-phonon modes $(1,6)$ and $(1,6)^{\prime}$. The modes $(1,2)^{\prime},(2,9)^{\prime},(1,4)^{\prime}$, and $(2,7)$ are also identified in Fig. 3(b), but they are of a very small spectral weight, even for the highest density. In Fig. 3(a), we see the coupled extra mode $(3,9)^{\prime}$, related to electrons in the third subband, gaining spectral weight when the third subband is getting occupied. This happens for $N_{e}$ $=1.9 \times 10^{6} \mathrm{~cm}^{-1}$. For lower densities, this mode is also seen, but with smaller intensity.

The inset shows the energy position of all peaks as a function of total electron density $N_{e}$. We point out that the coupled intersubband plasmon-phonon modes $(1,6)$ and $(2,7)$ shift to higher frequencies as the density increases due to the enhancement of the depolarization shift. At the same time, the complementary extra modes $(n, m)^{\prime}$, which lie below $\omega_{L O}$ shift instead to lower frequencies. This sort of dependence is due to the strong electron-phonon coupling occurring in this region. It can be interpreted as a signature of the electronphonon interaction.

We further explore this system by showing in Fig. 4 the $\omega$ position (open dot curves) of the most weighted peaks in (a) $S^{\text {even }}(q, \omega)$ and (b) $S^{\text {odd }}(q, \omega)$ as a function of the temperature. We take the electron density $N_{e}=1.0 \times 10^{6} \mathrm{~cm}^{-1}$ and the wave vector $q=0.05 k_{0}$. As the temperature increases, the electron density in the first (second) subband decreases (in- creases). As a consequence, the peaks of the intersubband coupled modes $(1, n)((2, n))$ related to the first (second) subband shift to lower (higher) frequencies due to the decrease (increase) of the depolarization shift. Such a change is mainly induced by the redistribution of the electron density in the different subbands. However, the extra modes $(1, n)^{\prime}$ and $(2, n)^{\prime}$, which are seen in the insets and appear below $\omega_{L O}$, present the opposite behavior. This is the same signature which appears in the previous figure. The clearer regions indicate the survival of the intersubband coupled modes $(1, n)$ for room temperatures.

For the sake of completeness, we compare, in Fig. 5, the dispersion relations at $T=300 \mathrm{~K}$ for two different electron densities. We show in Figs. 5(a) and 5(b) the intersubband coupled modes $(1, n)$ for $N_{e}=1.0 \times 10^{6} \mathrm{~cm}^{-1}$, while Figs. 5(c) and 5(d) show the same for $N_{e}=2.2 \times 10^{6} \mathrm{~cm}^{-1}$. Spectral weight intensities decrease as the wave vector $q$ increases, but they are still clearly noticeable at $T=300 \mathrm{~K}$ (red regions) for both densities.

\section{SUMMARY}

In summary, we have mapped out the $\omega-q$ plane concerning coupled intersubband plasmon-phonon modes at room temperatures in multisubband Q1D quantum wires based on polar semiconductor structures. We analyzed them by looking at the peaks of the spectral weight function. We studied the temperature dependence of the extra coupled plasmonphonon modes which arise as a consequence of the electronphonon interaction. We also studied them as a function of the electron density in the vicinity of longitudinal-optical phonon frequency $\omega_{L O}$. We remarkably found coupled intersubband plasmon-phonon modes related to the first subband surviving up to temperatures as high as $300 \mathrm{~K}$.

\section{ACKNOWLEDGMENTS}

The author thanks G.-Q. Hai for helpful discussions. This work was supported by FAPESP, the research foundation agency of the state of São Paulo, Brazil.
${ }^{1}$ A. R. Goñi, A. Pinczuk, J. S. Weiner, J. M. Calleja, B. S. Dennis, L. N. Pfeiffer, and K. W. West, Phys. Rev. Lett. 67, 3298 (1991).

${ }^{2}$ S. Das Sarma and W. Y. Lai, Phys. Rev. B 32, 1401 (1985); Q. P. Li and S. Das Sarma, ibid. 43, 11768 (1991); W.-M. Que and G. Kirczenow, ibid. 39, 5998 (1989).

${ }^{3}$ G. Abstreiter, M. Cardona, and A. Pinczuk, in Light Scattering in Solids IV, edited by M. Cardonna and G. Güntherodt (SpringerVerlag, Berlin, 1984), p. 36; G. Lermann, T. Bischof, A. Materny, W. Kiefer, T. Kummell, G. Bacher, A. Forchel, and G. Landwehr, J. Appl. Phys. 81, 1446 (1997); M. D. Efremov, V. A. Volodin, V. A. Sachkov, V. V. Preobrazhenskii, B. R. Semyagin, D. V. Marin, R. S. Matvienko, N. N. Ledentsov, I. P. Soshnikov, D. Litvinov, A. Rosenauer, and D. Gerthsen, Physica E (Amsterdam) 23, 461 (2004); and for a latest review see
D.-W. Wang, A. J. Millis, and S. Das Sarma, cond-mat/0406121 (unpublished).

${ }^{4}$ L. Zheng and S. Das Sarma, Phys. Rev. B 54, 2751 (1996).

${ }^{5}$ A. Mlayah, R. Carles, E. Bedel, and A. Muñoz-Yagüe, Appl. Phys. Lett. 62, 2848 (1993); J. Appl. Phys. 74, 1072 (1993); G.-Q Hai, N. Studart, and G. E. Marques, Phys. Rev. B 57, 2276 (1998).

${ }^{6}$ I. Keck, S. Schmult, and W. Wegscheider, Phys. Rev. B 67, 125312 (2003), and references therein.

${ }^{7}$ M. R. S. Tavares, G.-Q. Hai, F. M. Petters, and N. Studart, Phys. Rev. B 68, 140504(R) (2003); M. R. S. Tavares, G.-Q. Hai, and G. E. Marques, ibid. 68, 165304 (2003).

${ }^{8}$ G. Coli and R. Cingolani, Solid State Commun. 110, 293 (1999).

${ }^{9}$ L. Wendler, R. Haupt, and R. Pechstedt, Phys. Rev. B 43, 14669 (1991); L. Wendler, R. Haupt, and V. G. Grigoryan, Physica B 167, 113 (1990). 
${ }^{10}$ E. H. Hwang and S. Das Sarma, Phys. Rev. B 52, R8668 (1995)

${ }^{11}$ For a review, see G. D. Mahan, Many Particle Physics (Plenum, New York, 1981).

${ }^{12}$ G.-Q. Hai, N. Studart, and F. M. Peteers, Phys. Rev. B 52, 8363 (1995), and references therein.

${ }^{13}$ J.-B. Xia and G.-Q. Hai, Phys. Rev. B 65, 245326 (2002); M. R. S. Tavares, S. Das Sarma, and G.-Q. Hai, ibid. 63, 045324 (2001).

${ }^{14}$ M. R. S. Tavares and G.-Q Hai, Phys. Rev. B 61, 7564 (2000); S. Das Sarma and E. H. Hwang, Phys. Rev. Lett. 81, 4216 (1998).
${ }^{15}$ In the long wavelength limit, the intersubband modes in single wires turned out to be of similar a nature as the acoustic plasmon mode $\omega_{-}(q)$ appearing in biwire systems (Ref. 12). The acoustic mode represents charge density oscillating out of phase in the wires. Like intersubband modes, it also presents a frequency gap being finite at $q=0$. This gap appears only when the interwire tunneling (or first-excited subband occupancy) is allowed. The value of this gap is then directly dependent on the electron density in the second wire (or first-excited subband). 